\title{
Fractional boundaries for fluid spheres
}

\author{
S. Bayin \\ Department of Physics, Middle East Technical University, Ankara, Turkey \\ E. N. Glass and J. P. Krisch \\ Department of Physics, University of Michigan, Ann Arbor, Michigan 48109
}

(Received 4 July 2005; accepted 7 November 2005; published online 12 January 2006)

\begin{abstract}
A single Israel layer can be created when two metrics adjoin with no continuous metric derivative across the boundary. The properties of the layer depend only on the two metrics it separates. By using a fractional derivative match, a family of Israel layers can be created between the same two metrics. The family is indexed by the order of the fractional derivative. The method is applied to Tolman IV and V interiors and a Schwarzschild vacuum exterior. The method creates new ranges of modeling parameters for fluid spheres. A thin shell analysis clarifies pressure/ tension in the family of boundary layers. (C) 2006 American Institute of Physics.
\end{abstract}

[DOI: $10.1063 / 1.2158436]$

\section{INTRODUCTION}

There is long-standing interest in fluid sphere solutions, largely because of their astrophysical implications. An astrophysical model is often an interior fluid sphere metric matched to a Schwarzschild vacuum or Kottler exterior across a bounding surface. The standard technique matches metric functions and extrinsic curvatures on the boundary. When the extrinsic curvatures do not match, an Israel boundary layer ${ }^{1,2}$ can be created. The layer depends only on the properties of the two bounding metrics. Methods that will create a family of surface layers between the bounds could prove useful in exploring models of spheres with variable crusts. One way of creating variable surface layers is to modify the boundary conditions at the fluid-vacuum interface.

While an extrinsic curvature match is the boundary condition currently most used, there are three types of boundary conditions that have been used to match analytic solutions across non-null boundaries. The three methods have been discussed by Bonnor and Vickers, ${ }^{3}$ and they all involve derivatives of the metric functions. The boundary conditions can be generalized by broadening the idea of derivatives to include fractional derivatives. ${ }^{4,5}$

There are two simple ways to proceed with the generalization. The first is to assume a straight fractional derivative match on the boundary metrics and then to use the fractional relations in the usual formalism for the boundary stress energy. This would be a generalization of the Lichnerowicz boundary condition. It would not generalize the extrinsic curvature to fractional values. The second would be to use fractional derivatives to define a fractional extrinsic curvature and then use it to define a fractional boundary layer. This would be a generalization of the usual Lie derivative to fractional values. The use of fractional calculus is motivated by the possible fractional nature of the growth processes forming the boundary layer. Fractional transport processes are one of the main areas of application for fractional calculus, and boundary layers formed by these processes could reflect this fractional formation process.

Beyond the fractional generalizations of techniques and tensor functions, one must consider the various definitions of fractional differentiation. Use of fractional calculus in diverse areas of physics has increased enormously since fractional derivatives were first considered by Leibnitz and L'Hospital ${ }^{4}$ in 1695. Many different definitions have been proposed for different applications. In this article we use the Caputo form of the Riemann-Liouville and Weyl definitions. The Caputo derivative is an integral transform of the regular partial derivative and preserves the zero fractional 
derivative of a constant. While considering generalizations of relativistic gravity to include fractional calculus, the different definitions must be explored to determine their applicability.

This work has two goals: first to develop a variable layer model that could be applied to astrophysical problems, and second to better understand the role that fractional derivatives might play within a general relativistic framework. In this paper we will apply the first method and use fractional derivatives to create a family of Israel boundary layers between two bounding metrics. The family is parametrized by the order of the fractional derivative and may be used to model fluid spheres with variable crusts. Even when a regular derivative match is possible, the fractional match will broaden the parameter ranges for the fluid interior.

In the next section we discuss the metrics and describe the boundary layer. In the third section several models are considered: the Misner-Zapolsky (MZ) solution, ${ }^{6,7}$ and Tolman's solutions IV and $\mathrm{V}^{8}$ The thin shell pressure balance is treated in the fourth section and some details of the fractional match are discussed in Sec. V. Details of the fractional derivatives and the standard fluid sphere formalism are given in the Appendices.

\section{THEORETICAL FRAMEWORK}

\section{A. The space-time}

The two regions to be considered are covered by an exterior Schwarzschild solution bounding an interior spherical fluid. The metrics are, with functions $\psi_{\mathrm{Sch}}=1-2 m_{0} / y, \nu(r), \lambda(r), H(r)$ :

$$
\begin{gathered}
\text { Exterior: } g_{\alpha \beta}^{\text {Sch }} d x^{\alpha} d x^{\beta}=-\psi_{\text {Sch }} d t^{2}+\psi_{\text {Sch }}^{-1} d y^{2}+y^{2} d \Omega^{2}, \\
\text { Interior: } g_{\alpha \beta}^{\text {fluid }} d x^{\alpha} d x^{\beta}=-e^{\nu} d \tau^{2}+e^{\lambda} d r^{2}+H^{2} d \Omega^{2} .
\end{gathered}
$$

The bounding surface is located at $y=y_{0}$ in the exterior and $r=R_{0}$ in the interior. The corresponding normals to the surface are

$$
\begin{gathered}
\text { Exterior: } n_{\mu}^{E} d x^{\mu}=\psi_{\mathrm{Sch}}^{-1 / 2} d y, \\
\text { Interior: } n_{\mu}^{I} d x^{\mu}=e^{\lambda / 2} d r .
\end{gathered}
$$

Fractional derivatives leave these metrics unchanged. Our fractional extension provides a crust layer between the interior and exterior metrics.

\section{B. Matching conditions}

On the boundary, the metric match conditions are

$$
\begin{gathered}
\left(1-2 m_{0} / R_{0}\right)=e^{\nu\left(R_{0}\right)}, \\
R_{0}=H\left(R_{0}\right) .
\end{gathered}
$$

The second matching condition is the extrinsic curvature match, $K_{b}^{a}$, on the bounding surface. If the curvatures do not match, an Israel boundary layer is created. The stress-energy content of the Israel layer is constructed from the mismatch in the extrinsic curvatures. The stress-energy of the boundary layer is ${ }^{2}$

$$
-8 \pi S_{b}^{a}=\left\langle K_{b}^{a}\right\rangle-\langle K\rangle g_{b}^{a} .
$$

Here $K=K_{a}^{a}$. The stress-energy components on the boundary are

$$
-8 \pi S_{0}^{0}=\left[\left\langle K_{0}^{0}\right\rangle-\left\langle K_{0}^{0}+2 K_{\theta}^{\theta}\right\rangle g_{0}^{0}\right]=-2\left\langle K_{\theta}^{\theta}\right\rangle,
$$




$$
-8 \pi S_{\theta}^{\theta}=-8 \pi S_{\phi}^{\phi}=\left[\left\langle K_{\theta}^{\theta}\right\rangle-\left\langle K_{0}^{0}+2 K_{\theta}^{\theta}\right\rangle g_{\theta}^{\theta}\right]=-\left[\left\langle K_{\theta}^{\theta}\right\rangle+\left\langle K_{0}^{0}\right\rangle\right],
$$

and the stress-energy of the boundary is

$$
\begin{gathered}
8 \pi S_{0}^{0}=\frac{1}{\left(g_{y y}^{E}\right)^{1 / 2}} \frac{g_{\theta \theta, y}^{E}}{g_{\theta \theta}^{E}}-\frac{1}{\left(g_{r r}^{I}\right)^{1 / 2}} \frac{g_{\theta \theta, r}^{I}}{g_{\theta \theta}^{I}} \\
8 \pi S_{\theta}^{\theta}=8 \pi S_{\phi}^{\phi}=\frac{1}{2}\left[\frac{1}{\left(g_{y y}^{E}\right)^{1 / 2}} \frac{g_{\theta \theta, y}^{E}}{g_{\theta \theta}^{E}}-\frac{1}{\left(g_{r r}^{I}\right)^{1 / 2}} \frac{g_{\theta \theta, r}^{I}}{g_{\theta \theta}^{I}}\right]+\frac{1}{2}\left[\frac{1}{\left(g_{y y}^{E}\right)^{1 / 2}} \frac{g_{00, y}^{E}}{g_{00}^{E}}-\frac{1}{\left(g_{r r}^{I}\right)^{1 / 2}} \frac{g_{00, r}^{I}}{g_{00}^{I}}\right] .
\end{gathered}
$$

\section{Match of fractional derivatives}

The stress-energy of the Israel layer is evaluated on the boundary between the interior and exterior metrics. The actual finite thickness boundary layer is modeled by the single bounding surface at $r=R_{0}$. The stress-energy content is governed by regular derivatives of the metric functions. The metric match coupled with some derivative match of the metric on the layer, sets relations between the parameters of the interior and exterior solutions. With the usual extrinsic curvature or other derivative matches, the properties of the layer are set by the parameters of the bounding metric. With a fractional match, the order of the fractional derivative enters along with the other parameters and a family of fractional boundary layers is created. The fluid sphere examples considered in this paper have boundary metrics of the form

$$
d s^{2}=-F(r) d t^{2}+r^{2} d \Omega^{2} .
$$

The fractional match is applied only to the differing part of the Israel layer metric, the $g_{00}$ metric potential. The actual calculation of the fractional derivatives involves a choice of definition. We use the Caputo definition (see Appendix A) with the $\left(0 \leqslant r \leqslant R_{0}\right)$ Riemann-Liouville limits for the interior and the $\left(R_{0} \leqslant r \leqslant \infty\right)$ Weyl limits for the exterior. The limits themselves, as well as the choice of different limits for interior and exterior derivatives, reflect the nonlocality of the fractional derivative operation. Nonlocality in fractional time derivatives is an expression of system memory. ${ }^{9}$ It has proven especially useful in modeling jump processes with long wait times. ${ }^{10}$ Similarly, spatial nonlocality implies that the derivative on the boundary depends on values away from the boundary; fractional spatial derivatives have been useful in modeling processes with very large jump distances. ${ }^{11}$ When the jump distance depends on the jump time, fractional time and spatial derivatives enter into the transport equations. ${ }^{12}$ The examples discussed here are static, but the structure of the boundary layer could reflect the transport process. The fractional matching condition is

$$
\frac{1}{\Gamma(n-\alpha)} \int_{0}^{R_{0}} \frac{d^{n} F_{I}(x) / d x^{n}}{(r-x)^{\alpha-n+1}} d x=\frac{(-1)^{n-1}}{\Gamma(n-\alpha)} \int_{R_{0}}^{\infty} \frac{d^{n} F_{E}(x) / d x^{n}}{(x-r)^{\alpha-n}} d x,
$$

and is applied at $r=R_{0}$. We note that the single layer at $r=R_{0}$ only approximates a boundary of finite thickness and that using a nonlocal operator might be a better approximation to the actual match over a finite thickness than the usual derivative match over a zero thickness surface.

In the next sections, we apply the formalism to Tolman IV and V solutions.

\section{MODEL CALCULATIONS}

\section{A. Tolman's Solution V}

\section{The solution}

We consider a parametrization of Tolman's Vth solution. ${ }^{8,13}$ The metric, with constants $n$ and $C$, is 


$$
d s^{2}=-\left(r / r_{0}\right)^{N_{1}} d t^{2}+a\left(1-a C r^{2+b}\right)^{-1} d r^{2}+r^{2} d \Omega^{2} .
$$

The parameters formed from $n$ are

$$
\begin{gathered}
N_{1}=4 n /(1+n), \quad N_{2}=1+6 n+n^{2}, \\
a=\frac{N_{2}}{(1+n)^{2}}, \quad b=\frac{N_{1}(1-n)}{(1+3 n)} .
\end{gathered}
$$

The interior density and pressure for this solution are

$$
\begin{gathered}
8 \pi \rho=\left(\frac{4 n}{N_{2}}\right) \frac{1}{r^{2}}+C(3+b) r^{b}, \\
8 \pi P=\left(\frac{4 n^{2}}{N_{2}}\right) \frac{1}{r^{2}}-C \frac{(1+5 n)}{1+n} r^{b} .
\end{gathered}
$$

For $C=0$, the solution reduces to the MZ solution. ${ }^{6,7,15}$ This solution was originally used to describe neutron star models with equation of state $P=n \rho$. The solution with $C=0$ does not admit a zero-pressure boundary; the $C \neq 0$ solution does. Both solutions are singular at the origin and are used to represent an ultrahigh density core. The MZ solution, lacking a vacuum boundary, is generally matched to a gaseous envelope. It may be more realistic in some cases to match these solutions to a crust with surface stresses.

For $C \neq 0$, the zero-pressure boundary, $R_{z}$, relates constants $C$ and $n$,

$$
C=\frac{4 n^{2}(1+n)}{(1+5 n) N_{2}} \frac{1}{R_{z}^{2+b}} .
$$

Substituting for $C$, the pressure and density can be written as

$$
\begin{gathered}
8 \pi P_{C \neq 0}=\frac{4 n^{2}}{N_{2}}\left(\frac{1}{r^{2}}-\frac{r^{b}}{R_{z}^{2+b}}\right), \\
8 \pi \rho_{C \neq 0}=\frac{4 n}{N_{2}}\left(\frac{1}{r^{2}}\right)+\frac{4 n^{2}}{N_{2}} \frac{(n+3)}{(1+3 n)}\left(\frac{r^{b}}{R_{z}^{2+b}}\right) .
\end{gathered}
$$

The condition $P_{C \neq 0} \geqslant 0$ requires

$$
R_{0} \leqslant R_{z}
$$

Fractional matching will allow a broader family of sphere sizes. Below, we graph values for the case $n=1 / 3$. For $C=0$, there is no zero-pressure boundary and no constraint.

\section{Matching conditions}

The matching conditions are the same for any $C$ value. Matching the interior metric to vacuum Schwarzschild, we find

$$
1-2 m_{0} / R_{0}=\left(R_{0} / r_{0}\right)^{N_{1}}
$$

[recall $\left.N_{1}=4 n /(1+n)\right]$,

$$
\frac{2 m_{0}}{R_{0}^{1+\alpha}} \Gamma(1+\alpha)=\left(\frac{R_{0}}{r_{0}}\right)^{N_{1}}\left(\frac{N_{1}}{R_{0}^{\alpha}}\right) \frac{\Gamma\left(N_{1}\right)}{\Gamma\left(N_{1}+1-\alpha\right)} .
$$

Combining the two relations, we find that the scaled radius of the interior is 


$$
\frac{R_{0}}{2 m_{0}}=1+\frac{\Gamma(1+\alpha) \Gamma\left(N_{1}+1-\alpha\right)}{\Gamma\left(1+N_{1}\right)} .
$$

Note that the boundary radius is always greater than $2 m_{0}$. For $\alpha<1$, there are no limits imposed by Eq. (14). For $\alpha \geqslant 1$ we require

$$
1+N_{1}>\alpha .
$$

The metric parameter $r_{0}$ is described by

$$
\left(\frac{r_{0}}{R_{0}}\right)^{N_{1}}=1+\frac{\Gamma\left(1+N_{1}\right)}{\Gamma(1+\alpha) \Gamma\left(N_{1}+1-\alpha\right)} .
$$

The sizes of the fractional spheres are discussed in Sec. V.

\section{The crust stress-energy}

The stress-energy of the crust for general $C$ is, with $\gamma_{0}:=\sqrt{1-2 m_{0} / R_{0}}$,

$$
\begin{gathered}
8 \pi S_{0}^{0}=\frac{2}{R_{0}}\left[\gamma_{0}-(1+n) N_{2}^{-1 / 2} \sqrt{1-a C R_{0}^{2+b}}\right] \\
8 \pi S_{\theta}^{\theta}=8 \pi S_{\phi}^{\phi}=\frac{1}{2 R_{0}}\left[\gamma_{0}+1 / \gamma_{0}-(1+n)\left(2+N_{1}\right) N_{2}^{-1 / 2} \sqrt{1-a C R_{0}^{2+b}} .\right.
\end{gathered}
$$

For $C \neq 0$, the boundary layer has a stress-energy content [recall $N_{1}=4 n /(1+n), N_{2}=1+6 n+n^{2}$ ],

$$
\begin{gathered}
8 \pi S_{0}^{0}=\frac{2}{R_{0}}\left[\gamma_{0}-(1+n) N_{2}^{-1 / 2} \sqrt{1-\frac{n N_{1}}{(1+5 n)}\left(R_{0} / R_{z}\right)^{2+b}}\right], \\
8 \pi S_{\theta}^{\theta}=8 \pi S_{\phi}^{\phi}=\frac{1}{2 R_{0}}\left[\gamma_{0}+1 / \gamma_{0}-2(1+3 n) N_{2}^{-1 / 2} \sqrt{1-\frac{n N_{1}}{(1+5 n)}\left(R_{0} / R_{z}\right)^{2+b}}\right] .
\end{gathered}
$$

For $C=0$ the fluid energy density and stress are

$$
\begin{aligned}
8 \pi S_{0}^{0} & =\left(2 / R_{0}\right)\left[\gamma_{0}-(1+n) N_{2}^{-1 / 2}\right] \\
& =\left(2 / R_{0}\right)\left[\left(R_{0} / r_{0}\right)^{2 n /(1+n)}-(1+n) N_{2}^{-1 / 2}\right], \\
8 \pi S_{\theta}^{\theta} & =8 \pi S_{\phi}^{\phi}=\left(1 / R_{0}\right)\left[\left(1-m_{0} / R_{0}\right) / \gamma_{0}-(1+3 n) N_{2}^{-1 / 2}\right],
\end{aligned}
$$

and describe a much richer modeling environment.

\section{B. Tolman's Solution IV}

\section{Metric and stress-energy}

This solution describes an object with finite central presssure and density. A stiff fluid core is not possible in this model. The interior metric for this solution is, with constants $A, B$, and $C$,

$$
d s^{2}=-B^{2}\left(1+r^{2} / A^{2}\right) d t^{2}+\frac{1+2 r^{2} / A^{2}}{\left(1-r^{2} / C^{2}\right)\left(1+r^{2} / A^{2}\right)} d r^{2}+r^{2} d \Omega^{2} .
$$

The interior density and pressure are

$$
8 \pi \rho=\frac{1}{A^{2}}\left[\frac{1+3\left(A^{2} / C^{2}+r^{2} / C^{2}\right)}{1+2 r^{2} / A^{2}}+2 \frac{1-r^{2} / C^{2}}{\left(1+2 r^{2} / A^{2}\right)^{2}}\right],
$$




$$
8 \pi P=\frac{1}{A^{2}}\left[\frac{1-\left(A^{2} / C^{2}+3 r^{2} / C^{2}\right)}{1+2 r^{2} / A^{2}}\right] .
$$

Constants $A$ and $C$ can be expressed in terms of the central fluid values. We have

$$
\begin{gathered}
8 \pi \rho_{c}=\frac{3}{A^{2}}\left[1+A^{2} / C^{2}\right], \\
8 \pi P_{c}=\frac{1}{A^{2}}\left[1-A^{2} / C^{2}\right], \\
A^{2}=\frac{2}{8 \pi\left(\rho_{c} / 3+P_{c}\right)}, \\
C^{2}=\frac{2}{8 \pi\left(\rho_{c} / 3-P_{c}\right)} .
\end{gathered}
$$

Note that the central fluid equation of state (EOS) is constrained: $P_{c}<\rho_{c} / 3$. The zero-pressure boundary that occurs in the regular derivative match has size

$$
R_{z}^{2}=C^{2} / 3-A^{2} / 3
$$

\section{Metric match}

The match to vacuum Schwarzschild provides

$$
B^{2}\left(1+R_{0}^{2} / A^{2}\right)=1-2 m_{0} / R_{0} \text {. }
$$

The fractional match is

$$
\begin{gathered}
\frac{B^{2}}{A^{2}} \frac{1}{R_{0}^{\alpha-2}} \frac{1}{\Gamma(3-\alpha)}=\frac{m_{0}}{R_{0}^{1+\alpha}} \Gamma(1+\alpha), \\
B^{2} R_{0}^{3}=A^{2} m_{0} \Gamma(3-\alpha) \Gamma(1+\alpha) .
\end{gathered}
$$

Combining with the metric match, we obtain

$$
\begin{gathered}
A^{2}=R_{0}^{2} \frac{\left(R_{0} / m_{0}\right)-[2+\Gamma(3-\alpha) \Gamma(1+\alpha)]}{\Gamma(3-\alpha) \Gamma(1+\alpha)}, \\
B^{2}=\frac{m_{0}}{R_{0}}\left[\frac{R_{0}}{m_{0}}-[2+\Gamma(3-\alpha) \Gamma(1+\alpha)]\right], \\
R_{0} / m_{0}>2+\Gamma(3-\alpha) \Gamma(1+\alpha) .
\end{gathered}
$$

The boundary size depends on the central EOS as well as the order of the fractional derivative:

$$
\left(\frac{R_{0}}{m_{0}}\right)^{3}-\left(\frac{R_{0}}{m_{0}}\right)^{2}[2+\Gamma(3-\alpha) \Gamma(1+\alpha)]-\frac{\Gamma(3-\alpha) \Gamma(1+\alpha)}{4 \pi m_{0}^{2}\left(P_{c}+\rho_{c} / 3\right)}=0 .
$$




\section{Crust stress-energy}

We introduce scaled parameters $r_{A}^{2}:=R_{0}^{2} / A^{2}, r_{C}^{2}:=R_{0}^{2} / C^{2}$, and $r_{z}^{2}:=R_{z}^{2} / A^{2}$.

$$
\begin{aligned}
8 \pi S_{0}^{0} & =\frac{1}{\left(g_{y y}^{E}\right)^{1 / 2}} \frac{g_{\theta \theta, y}^{E}}{g_{\theta \theta}^{E}}-\frac{1}{\left(g_{r r}^{I}\right)^{1 / 2}} \frac{g_{\theta \theta, r}^{I}}{g_{\theta \theta}^{I}}=\frac{2}{R_{0}}\left[\sqrt{1-2 m_{0} / R_{0}}-\sqrt{\frac{\left(1-r_{C}^{2}\right)\left(1+r_{A}^{2}\right)}{1+2 r_{A}^{2}}}\right] \\
& =\frac{2}{R_{0}} \sqrt{1+r_{A}^{2}}\left[B-\frac{A}{C} \sqrt{\frac{1+3 r_{z}^{2}-r_{A}^{2}}{1+2 r_{A}^{2}}}\right], \\
8 \pi S_{\theta}^{\theta} & =8 \pi S_{\phi}^{\phi}=\frac{1}{2}\left[\frac{1}{\left(g_{y y}^{E}\right)^{1 / 2}} \frac{g_{\theta \theta, y}^{E}}{g_{\theta \theta}^{E}}-\frac{1}{\left(g_{r r}^{I}\right)^{1 / 2}} \frac{g_{\theta \theta, r}^{I}}{g_{\theta \theta}^{I}}\right]+\frac{1}{2}\left[\frac{1}{\left(g_{y y}^{E}\right)^{1 / 2}} \frac{g_{00, y}^{E}}{g_{00}^{E}}-\frac{1}{\left(g_{r r}^{I}\right)^{1 / 2}} \frac{g_{00, r}^{I}}{g_{00}^{I}}\right] \\
= & \frac{1}{R_{0}} \sqrt{1+r_{A}^{2}}\left[B-\frac{A}{C} \sqrt{\frac{1+3 r_{z}^{2}-r_{A}^{2}}{1+2 r_{A}^{2}}}\right]+\frac{1}{R_{0} \sqrt{1+r_{A}^{2}}}\left[\frac{m_{0} / R_{0}}{B}-\left(r_{A}^{2}\right) \sqrt{\frac{1-r_{C}^{2}}{1+2 r_{A}^{2}}}\right] .
\end{aligned}
$$

Some examples of radius variation and crust stress energy are given in Sec. V.

\section{EQUILIBRIUM IN THE PRESENCE OF SURFACE STRESSES}

\section{A. Stress-energy}

The Israel layer is the zero-thickness idealization of a bounding layer with finite thickness, $d$. The physical crust runs from an outer boundary $R_{0}$ to an interior fluid boundary $R_{i}$ with $d=R_{0}$ $-R_{i}$. We know that the interior fluid solutions will satisfy the ${ }^{22}$ Tolman-Oppenheimer-Volkov (TOV) equation. The Israel layers generated in this work are obtained by introducing a discontinuity in the derivative of $g_{00}$. The analog of the TOV equation for the layer, requiring that the solutions remain static, will provide relations among the model parameters. To develop the TOV analog for the layer, consider the general static spherical metric for an interior fluid with pressure $P$ and density $\rho$,

$$
d s^{2}=-e^{\nu} d t^{2}+e^{\lambda} d r^{2}+r^{2} d \Omega^{2} .
$$

The details of the field equations are given in Appendix B. The covariant derivative of the general energy-momentum tensor provides the conservation equation

$$
-\frac{\partial T_{r}^{r}}{\partial r}-\left(\frac{\nu^{\prime}}{2}+\frac{2}{r}\right) T_{r}^{r}+\left(\frac{\nu^{\prime}}{2}\right) T_{0}^{0}+\left(\frac{2}{r}\right) T_{\theta}^{\theta}=0 .
$$

For an isotropic fluid matched to vacuum, this is the usual TOV equation,

$$
\frac{\partial P}{\partial r}+\frac{\nu^{\prime}}{2}(P+\rho)=0 .
$$

It is the analog of this equation that we want for the Israel layer.

\section{B. The conservation equation over a limiting shell}

Consider a bounding shell that will approximate a thin surface layer. The central radius of the shell is $R$ with the outer boundary $R^{(+)}=R+d / 2$, and the inner interior fluid boundary at $R^{(-)}=R$ $-d / 2$. $d$ is the coordinate shell thickness. In the $d \rightarrow 0$ limit, $R \rightarrow R_{0}$. A general stress-energy $T_{j}^{i}$ can be related to a surface stress-energy $S_{b}^{a}$ by $^{2}$

$$
T_{j}^{i}=\delta(l) S_{b}^{a} e_{a}^{i} e_{j}^{b},
$$

where $e_{a}^{i}$ is a tangent vector to the shell, and $l$ the proper distance along a radial geodesic, $l$ $=e^{\lambda / 2} d$. The shell stress-energy has a perfect fluid analog, 


$$
\begin{gathered}
S^{i j}=\sigma U^{i} U^{j}+\tau\left(h^{i j}+U^{i} U^{j}\right), \\
h^{i j}=g^{i j}-n^{i} n^{j}, \\
n^{i}=\left(0, e^{-\lambda}, 0,0\right),
\end{gathered}
$$

where $S_{0}^{0} / c^{2}=-\sigma\left(\mathrm{g} / \mathrm{cm}^{2}\right)$ and $S_{\theta}^{\theta}= \pm \tau($ dynes $/ \mathrm{cm})$. Following Poisson ${ }^{2}$, we take $l=0$ on the hypersurface defined by $R$, with $l$ negative for $r<R$ and positive on the vacuum side, $r>R$. The $T_{r}^{r}$ content of the shell can be described using a Heaviside function, $\Theta(l)$, as

$$
T_{r}^{r}=\Theta(l) T_{r}^{(+) r}+\Theta(-l) T_{r}^{(-) r}+\delta(l) S_{r}^{r} .
$$

The last term will be zero for the $2+1$ shell stress energy. Forming the derivative needed in the conservation equation, we have

$$
\frac{\partial T_{r}^{r}}{\partial r}=\delta(l) \frac{d l}{d r} T_{r}^{(+) r}+\Theta(l) \frac{\partial T_{r}^{(+) r}}{\partial r}-\delta(l) \frac{d l}{d r} T_{r}^{(-) r}+\Theta(-l) \frac{\partial T_{r}^{(-) r}}{\partial r} .
$$

In the $l \rightarrow 0$ limit we have

$$
\frac{\partial T_{r}^{r}}{\partial r}=-\lim _{l \rightarrow 0}\left[\delta(l) \frac{d l}{d r} T_{r}^{(-) r}\right]=-\lim _{l \rightarrow 0}\left[\delta(l) \mathrm{Pe}^{\lambda / 2}\right],
$$

where the first term is zero with no radial pressure on the outer boundary. The stress-energy function evaluated at the inner boundary is $P$ and $\lambda^{(-)}$is an interior metric function. Substituting into the conservation equation in the $l \rightarrow 0$ hypersurface limit, we have

$$
\mathrm{Pe}^{\lambda^{(-)} / 2}+\left(\nu^{\prime} / 2\right) S_{0}^{0}+\left(2 / R_{0}\right) S_{\theta}^{\theta}=0
$$

\section{Evaluating $\partial_{r} g_{00}$}

The derivative, $\nu^{\prime}$, on the hypersurface, can be written as a difference equation,

$$
\begin{aligned}
\nu^{\prime}(R) & =\frac{\nu(R+d / 2)-\nu(R-d / 2)}{(R+d / 2)-(R-d / 2)}=\frac{\nu(R+d / 2)-\nu(R)+\nu(R)-\nu(R-d / 2)}{(R+d / 2)-(R-d / 2)} \\
& =\frac{\nu(R+d / 2)-\nu(R)}{d}+\frac{\nu(R)-\nu(R-d / 2)}{d} .
\end{aligned}
$$

Expanding, we can write

$$
\nu(R \pm d / 2)=\nu(R) \pm \nu^{\prime}\left[R^{( \pm)}\right] \frac{d}{2}+\cdots
$$

Substituting in the thin shell limit, we have

$$
\nu^{\prime}(R) \approx \frac{\nu^{\prime}\left[R^{(+)}\right]+\nu^{\prime}\left[R^{(-)}\right]}{2} .
$$

The first term follows from the Schwarzschild metric match and the second is given in Appendix B. We have

$$
\nu^{\prime}(R) \approx\left(2 m / R^{2}+4 \pi R P\right)(1-2 m / R)^{-1},
$$

where we have identified the Schwarzschild mass parameter with the interior mass of the fluid. Substituting into Eq. (34), we have 


$$
-P(1-2 m / R)^{-1 / 2}=\left(m / R^{2}+2 \pi R P\right)(1-2 m / R)^{-1} S_{0}^{0}+(2 / R) S_{\theta}^{\theta},
$$

which describes the thin shell pressure balance. The classical limit of this equation follows from $c \rightarrow \infty$ and is

$$
P-\sigma \frac{m}{R^{2}}=\left(\frac{2}{R}\right)(-\tau) .
$$

If the fluid pressure at the interior boundary dominates, this can be interpreted as a tension in the shell, balancing the outward interior fluid pressure at the boundary minus the inward pressure due to the gravitational attraction of the shell by the interior fluid. If the shell mass term dominates, the stress in the boundary layer will be a pressure. In the next section we explore the stress-energy structure of the boundary layer, and will see parameter ranges with both layer tension and pressure.

\section{DETAILS OF THE FRACTIONAL MATCH}

\section{A. Sphere radii}

The sizes of the sphere are described by

$$
\begin{gathered}
\text { TolmanV: } \frac{R_{0}}{2 m_{0}}=1+\frac{\Gamma(1+\alpha) \Gamma(4 n /(1+n)+1-\alpha)}{\Gamma(1+4 n /(1+n))}, \\
\text { TolmanIV: }\left(\frac{R_{0}}{m_{0}}\right)^{3}-\left(\frac{R_{0}}{m_{0}}\right)^{2}[2+\Gamma(3-\alpha) \Gamma(1+\alpha)]-\frac{\Gamma(3-\alpha) \Gamma(1+\alpha)}{4 \pi m_{0}^{2}\left(P_{c}+\rho_{c} / 3\right)}=0 .
\end{gathered}
$$

The scaled boundary radius, $R_{0} / m_{0}$, for Tolman $\mathrm{V}$ is plotted as a function of alpha for various $n$ in Fig. 1.

The overall effect of the fractional match is to increase the range of sphere sizes for a given EOS. The largest differences are for low- $n$ fluids, where a very much smaller sphere radius is possible than for the zero-pressure match. The Buchdahl bound ${ }^{14}$ limits the ratio of $2 m_{0} / R_{0}$ for fluid spheres whose $g_{00}$ component is continuous across the boundary and whose density is

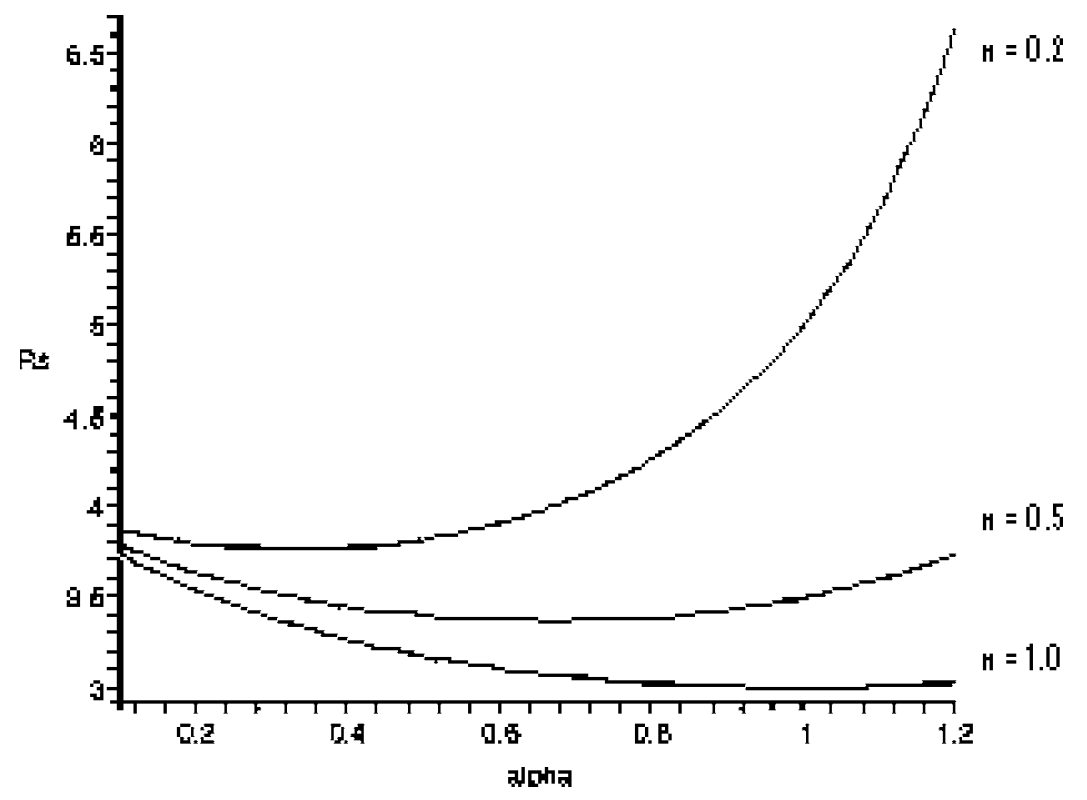

FIG. 1. Scaled radius versus fractional order. 
decreasing outward. We have matched fractional derivatives rather than first derivatives and it is not clear that the conditions of the Buchdahl bound are satisfied, but from Fig. 1, it is seen that the Buchdahl bound, $2 m_{0} / R_{0} \leqslant 8 / 9$, is not violated.

The radius for Tolman IV is a cubic root of Eq. (37), but some general description can be given. The modeling term in the equation is the denominator of the last term. Consider the factor

$$
c_{1} \sim 4 \pi m_{0}^{2} \rho_{c} / 3
$$

describing an object with mass $m_{0}=N m_{\odot}=N\left(2 \times 10^{30} \mathrm{~kg}\right)$, and central density and pressure of neutron star order, $\rho_{c} \sim 10^{17} \mathrm{~kg} / \mathrm{m}^{3}, P_{c} \sim 10^{33} \mathrm{Newton} / \mathrm{m}^{2}$. Numerical scaled radius values using these values are described in Table I for a range of $N$ and alpha values. For $N \sim 100$ or larger, the last term in the cubic is negligible and the radius is essentially given by the limiting value

$$
R_{0} / m_{0} \sim 2+\Gamma(3-\alpha) \Gamma(1+\alpha) .
$$

The masses for these radii are well out of the neutron star range. The reflection symmetry about $\alpha=1$ is the result of a product equivalence of the two gamma functions for paired alpha values, i.e., $\alpha=(0.6,1.4)$ give the same gamma function product. From Table I, it is clear that the low $N$ values have masses and radii of neutron star orders of magnitude. ${ }^{16}$ For example, for $N=1$, the radii are approximately $R_{0} \sim 11.44 m_{0} \sim 17 \mathrm{~km}$. Smaller central densities, describing more ordinary fluid objects, result in much larger fluid spheres. For a central density of $\rho_{c} \sim 10^{10} \mathrm{~kg} / \mathrm{m}^{3}$, the radii for $\alpha=1, \quad R_{0}(N)$ are $R_{0}(1)=2228.33 m_{0}, \quad R_{0}(10)=480.87 m_{0}, R_{0}(100)=104.39 m_{0}, R_{0}(1000)$ $=23.32 m_{0}$, with the values for larger and smaller $\alpha$ paired and increasing, just as for the larger central density.

TABLE I. $R_{0} / M_{0}$-Tolman IV- $\rho_{c} \sim 10^{17} \mathrm{~kg} / \mathrm{m}^{3}$.

\begin{tabular}{lcccc}
\hline \hline$\alpha$ & $N=1$ & $N=10$ & $N=100$ & $N=1000$ \\
\hline 0.2 & 13.24 & 4.41 & 3.55 & 3.54 \\
0.4 & 12.39 & 4.10 & 3.28 & 3.27 \\
0.6 & 11.85 & 3.91 & 3.12 & 3.11 \\
0.8 & 11.54 & 3.81 & 3.04 & 3.03 \\
1 & 11.44 & 3.78 & 3.01 & 3 \\
1.2 & 11.54 & 3.81 & 3.04 & 3.03 \\
1.4 & 11.85 & 3.91 & 3.12 & 3.11 \\
1.6 & 12.39 & 4.10 & 3.28 & 3.27 \\
\hline \hline
\end{tabular}

\section{B. Crust stress-energy}

Figures 2 and 3 describe the variation of the boundary energy density and pressure, in Tolman $\mathrm{V}, n=1 / 3$, as the size of the fluid sphere varies. Over a large part of the $R_{0} / R_{z}$ range, a crust tension contains the interior fluid, with the tension increasing in size as the fluid sphere becomes smaller, essentially acting to squeeze the fluid into smaller volumes. The results are similar for $C=0$. 


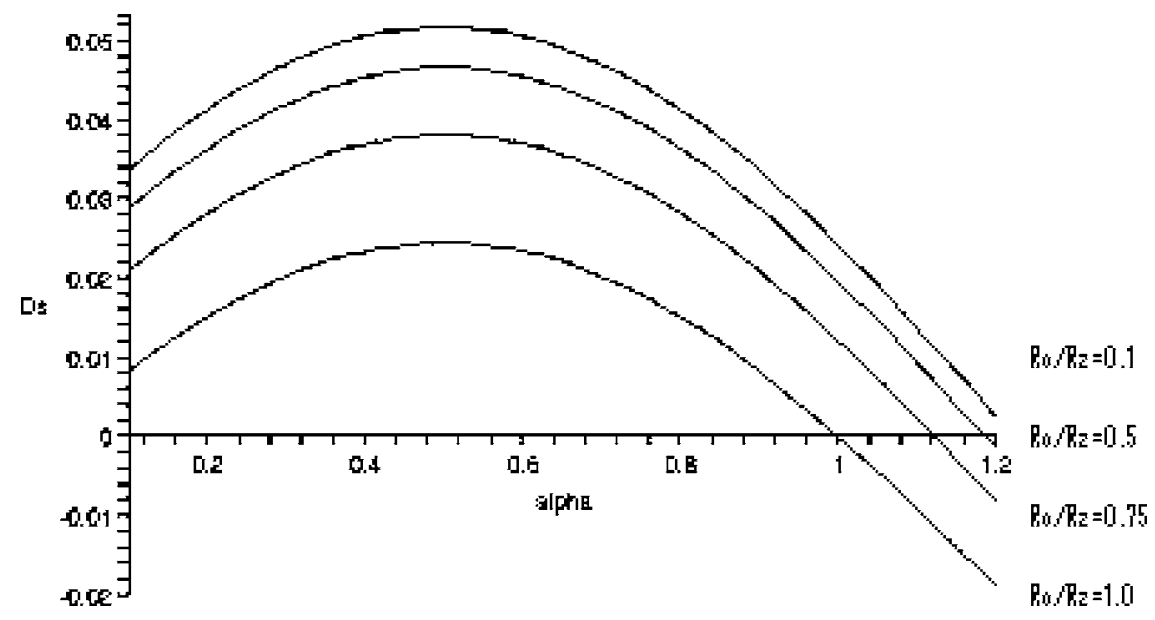

FIG. 2. $D s=-8 \pi m_{0} S_{0}^{0}$ versus fractional order.

For Tolman IV, the crust energy density is

$$
\begin{gathered}
8 \pi \sigma=\frac{2}{R_{0}}\left[-\sqrt{1-2 m_{0} / R_{0}}+\sqrt{\frac{\left(1-R_{0}^{2} / C^{2}\right)\left(1+R_{0}^{2} / A^{2}\right)}{1+2 R_{0}^{2} / A^{2}}}\right] \\
=\frac{2}{R_{0}}\left[-\sqrt{1-2 m_{0} / R_{0}}+\sqrt{\frac{\left[1-4 \pi R_{0}^{2} \rho_{c}(1 / 3-n)\right]\left[1+4 \pi \rho_{c} R_{0}^{2}(1 / 3+n)\right]}{1+R_{0}^{2} 8 \pi \rho_{c}(1 / 3+n)}}\right] .
\end{gathered}
$$

The modeling factor of importance is the term

$$
4 \pi \frac{R_{0}^{2}}{m_{0}^{2}} \rho_{c} m_{0}^{2}=3 c_{1} \frac{R_{0}^{2}}{m_{0}^{2}} .
$$

For nuclear central densities, $\rho_{c} \sim 10^{14} \mathrm{~g} / \mathrm{cm}^{3}$, this is

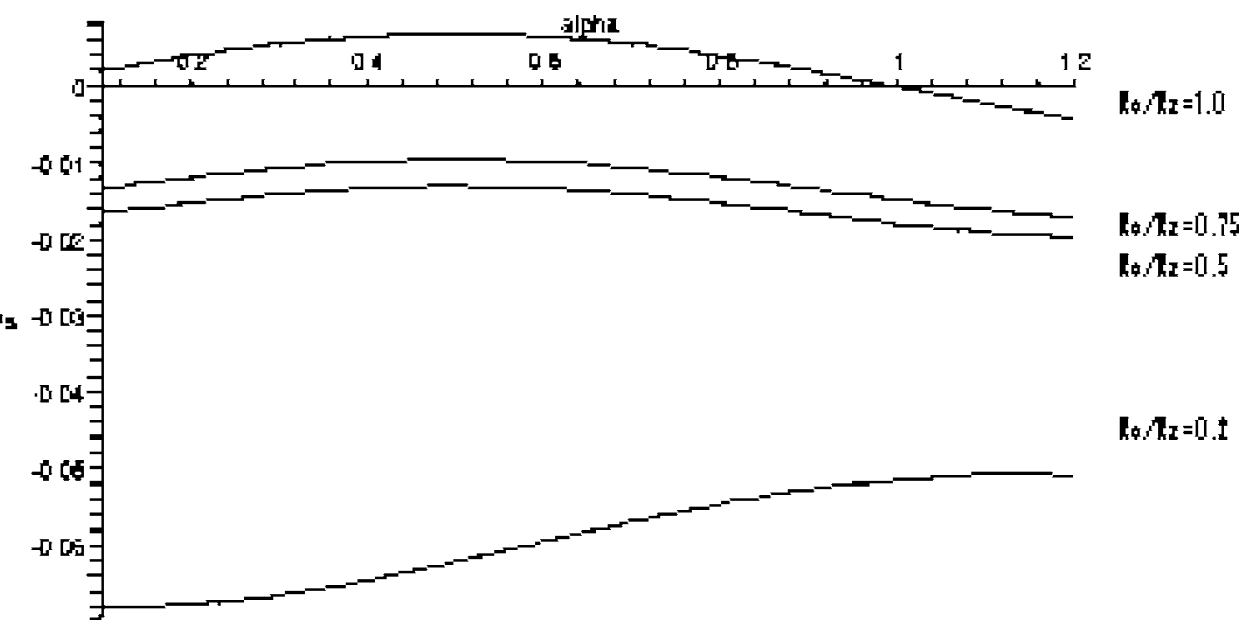

FIG. 3. $P s=8 \pi m_{0} S_{\vartheta}^{\vartheta}$ versus fractional order. 


$$
20.35 N^{2} \times 10^{-4} \frac{R_{0}^{2}}{m_{0}^{2}}
$$

In order to have real values, we require

$$
\begin{aligned}
& N=1, \quad R_{0} \sim 12 m_{0}, \quad[0.293 \times(1 / 3-n)]<1, \\
& N=10, \quad R_{0} \sim 4 m_{0}, \quad[3.26 \times(1 / 3-n)]<1, \\
& N=100, \quad R_{0} \sim 3 m_{0}, \quad[183 \times(1 / 3-n)]<1 .
\end{aligned}
$$

It is clear that the broadest range of central equations of state for nuclear central densities is for the lower mass objects. Higher mass objects require a central EOS very close to the 1/3 limit. For smaller values of the central density, the central EOS range is much broader. For a central density of $\rho_{c} \sim 10^{7} \mathrm{~g} / \mathrm{cm}^{3}$, the modeling factor is

$$
4 \pi \frac{R_{0}^{2}}{m_{0}^{2}} \rho_{c} m_{0}^{2}=20.35 N^{2} \times 10^{-11} \frac{R_{0}^{2}}{m_{0}^{2}},
$$

and for real values require

$$
\begin{gathered}
N=1, \quad R_{0} \sim 2228 m_{0}, \quad\left[0.10 \times 10^{-2}(1 / 3-n)\right]<1, \\
N=10, \quad R_{0} \sim 480 m_{0}, \quad\left[0.47 \times 10^{-2}(1 / 3-n)\right]<1, \\
N=100, \quad R_{0} \sim 104 m_{0}, \quad[0.022 \times(1 / 3-n)]<1, \\
N=1000, \quad R_{0} \sim 23 m_{0}, \quad[0.11 \times(1 / 3-n)]<1 .
\end{gathered}
$$

\section{CONCLUSION}

In this work we have examined a family of boundary layers created by matching fractional derivatives across a boundary. ${ }^{17,18}$ The boundary layers considered have structure that depends on the order of the fractional derivative. One of the reasons that fractional calculus may be important for boundary layers is the mechanism by which a boundary layer is formed. One of the possible ways to build a variable density crust is by a diffusive process; a process whose underlying cause is Brownian motion. This motion, as analyzed by statistical mechanics, involves diffusion, dissipation, and the fluctuation-dissipation theorem. The dynamical model of Brownian motion was provided by Langevin in 1908 using a stochastic differential equation. It seems apparent from the nature of randomness that such macroscopic stochastic equations are incompatible with the continuous and differentiable character of microscopic Hamiltonian dynamics. (Think of the conventional diffusion equation, with the diffusion process described by a second-order spatial derivative.) Therefore, the mathematical description rests on either ordinary analytical functions describing the dynamics, or on conventional differential operators describing the phase space evolution. The differentiable nature of the macroscopic picture is, in a sense, a natural consequence of microscopic randomness. This means that use can be made of ordinary differential calculations on the macroscopic scale, even if the microscopic dynamics are incompatible with ordinary calculus methods. On the other hand, in the case where a time scale separation between macroscopic and microscopic levels of description does not exist, the nondifferentiable nature of the microscopic dynamics is transmitted to the macroscopic level. Since fractional calculus has been shown to provide a good description for a range of diffusive processes, ${ }^{19}$ one might expect a boundary condition based on fractional calculus would reflect the fractional growth process. An 
example, given by Allegrini, Grigolini, and West, ${ }^{20}$ shows that a diffusion process generated by a fluctuation with no time scale at the macroscopic level generates a diffusion process well described by a fractional Laplacian.

While diffusion is a possible mechanism for generating a layer with structure, the method of generating the fractional family of layers is independent of the production mechanism and generates a family whose stress-energy and size depends on the order of the fractional derivative. The result is a much broader range in fluid sphere properties. For example, in Tolman V, a much larger range of spheres sizes can be described with the fractional layer than without, with the energy density of the layer decreasing as the size of the sphere increases. The $C \neq 0$ Tolman $\mathrm{V}$ spheres have a zero-pressure boundary solution. For spheres smaller than the zero-pressure sphere, the layer has a tension, while for spheres larger than the zero-pressure sphere, the layer has positive stress over much of the range of the fractional order. The fractional boundary could prove to be a valuable modeling tool in more realistic neutron star models.

The range of stress-energy in the fractional boundary layers implies differences in structure as a function of the fractional order. The differences in density could be modeled in several ways: for example, with different crust materials or different incomplete fluid coverings (tilings). ${ }^{21}$ The layer itself is a model of a thin crust and there could be differences in the geometry of the $2+1$ shells that fill the real crust. The interior fluid geometry and the exterior Schwarzschild vacuum do not have to match for a crust with finite thickness.

The models presented in this paper matched an integral transform of the regular derivative across a spatial boundary. It is not a fractional generalization of general relativity, but a fractional generalization of a boundary condition. The next step is to explore the range of fractional generalizations of other sets of boundary conditions and other derivative definitions to applicable spacetimes.

\section{APPENDIX A: DERIVATIVES}

\section{Regular}

For functions $f$ and $F$ continuous on $[a, b] \epsilon$ Reals,

$$
F(x):=\int_{a}^{x} f(t) d t
$$

$F(x)$ is differentiable such that $d F / d x=f$. The $n$th integer derivative is simply $d^{n} F / d x^{n}$. For example,

$$
\frac{d^{n}}{d x^{n}} x^{k}=\frac{k !}{(k-n) !} x^{k-n}
$$

With the gamma function this is

$$
\frac{d^{n}}{d x^{n}} x^{\alpha}=\frac{\Gamma(\alpha+1)}{\Gamma(\alpha-n+1)} x^{\alpha-n} .
$$

The gamma function, $\Gamma(z)$, is defined as

$$
\begin{gathered}
\Gamma(z)=\int_{0}^{\infty} e^{-t} t^{z-1} d t, \quad \Gamma(1 / 2)=\sqrt{\pi}, \quad \Gamma(1)=1, \\
\Gamma(n+1)=n \Gamma(n)=n !, \quad \text { for } n>0 .
\end{gathered}
$$




\section{Fractional}

\section{A. Riemann-Liouville}

The Riemann-Liouville definition for the $\alpha$ fractional derivative of $f(x)$ is, with $\alpha \geqslant 0$,

$$
D^{\alpha} f(x)=\frac{d^{\alpha}}{d x^{\alpha}} f(x):=\frac{1}{\Gamma(n-\alpha)} \frac{d^{n}}{d x^{n}} \int_{c}^{x} \frac{f(t)}{(x-t)^{\alpha-n+1}} d t,
$$

where $n$ is the smallest integer larger than $\alpha$ when it is fractional, that is, $n=[\alpha]+1$. In the $\alpha=1$ limit, the derivative produces the integer result. The constant $c$ in the limit of the integral is usually set to 0 (Riemann definition) or to $-\infty$ (Liouville definition). For example, the Riemann-Liouville derivative of $x^{k}$ for $\alpha \leqslant 1$ with $n=1$, we have

$$
\begin{aligned}
D^{\alpha} x^{k} & =\frac{1}{\Gamma(1-\alpha)} \frac{d}{d x} \int_{0}^{x} t^{k}(x-t)^{-\alpha} d t \\
& =\frac{1}{\Gamma(1-\alpha)} \frac{d}{d x} \int_{0}^{x} t^{k} x^{-\alpha}\left(1-\frac{t}{x}\right)^{-\alpha} d t \\
& =\frac{1}{\Gamma(1-\alpha)} \frac{d}{d x} \int_{0}^{1} w^{k} x^{-\alpha+1+k}(1-w)^{-\alpha} d w .
\end{aligned}
$$

Using the definition of the beta function,

$$
\int_{0}^{1} w^{p-1}(1-w)^{q-1} d w=\frac{\Gamma(p) \Gamma(q)}{\Gamma(p+q)},
$$

we have

$$
\begin{aligned}
D^{\alpha} x^{k} & =\frac{1}{\Gamma(1-\alpha)} \frac{d x^{-\alpha+1+k}}{d x} \frac{\Gamma(k+1) \Gamma(1-\alpha)}{\Gamma(k+2-\alpha)} \\
& =(1+k-\alpha) x^{k-\alpha} \frac{\Gamma(k+1)}{\Gamma(k+2-\alpha)} \\
& =x^{k-\alpha} \frac{\Gamma(k+1)}{\Gamma(k+1-\alpha)} .
\end{aligned}
$$

For $\alpha=1$, this is the usual result $D^{1} x^{k}=d x^{k-1}$. Note that, for $k=-1$, this operation fails. This is an example of one of the problems encountered in applying fractional derivatives to general relativity. Not all definitions of fractional derivatives work for all functions. The fractional derivative of $x^{k}$ for $\alpha \geqslant 1$ is identical to the fractional derivative for $\alpha \leqslant 1$. For this function, the derivative is continuous across the $\alpha=1$ boundary.

$$
\begin{aligned}
D^{\alpha} x^{k} & =\frac{1}{\Gamma(2-\alpha)} \frac{d^{2}}{d x^{2}} \int_{0}^{x} t^{k} x^{1-\alpha}\left(1-\frac{t}{x}\right)^{1-\alpha} d t \\
& =\frac{1}{\Gamma(2-\alpha)} \frac{d^{2}}{d x^{2}} \int_{0}^{1} w^{k} x^{k+2-\alpha}(1-w)^{1-\alpha} d w
\end{aligned}
$$




$$
=x^{k-\alpha} \frac{\Gamma(1+k)}{\Gamma(1+k-\alpha)} .
$$

One should note that the Riemann-Liouville fractional derivative of a constant is not zero.

\section{B. Caputo}

The Caputo derivative is the integral transform of the regular derivative and is found by moving the derivative in the Riemann-Liouville definition inside the integral to act on the function. We have

$$
D^{\alpha} f(x)=\frac{1}{\Gamma(n-\alpha)} \int_{0}^{x} \frac{\frac{d^{n}}{d t^{n}} f(t)}{(x-t)^{\alpha-n+1}} d t .
$$

Example: $f(x)=x^{b}, \beta \geqslant 0$,

$$
D^{\alpha}(x-a)^{k}=\frac{1}{\Gamma(n-\alpha)} \int_{0}^{x} \frac{\frac{d^{n}}{d t^{n}} t^{k}}{(x-t)^{\alpha-n+1}} d t .
$$

For $\alpha \leqslant 1, n=1$ we have

$$
D^{\alpha}(x-a)^{k}=\frac{1}{\Gamma(1-\alpha)} \int_{0}^{x} k t^{k-1}(x-t)^{-\alpha} d t=\frac{k x^{k-\alpha}}{\Gamma(1-\alpha)} \int_{0}^{1} w^{k-1}(1-w)^{-\alpha} d w=k x^{k-\alpha} \frac{\Gamma(k)}{\Gamma(k+1-\alpha)},
$$

which is identical to the Riemann-Liouville derivative for this function. This derivative also is not defined for $k=-1$. In general relativity, one of the space-times one would like to treat is vacuum Schwarzschild, but the Riemann-Liouville derivative will not give finite answers for the $1 / r$ structure. The derivative of $1 / r$ can be taken with the Weyl derivative.

\section{Weyl}

The Weyl derivative differs from the Riemann-Liouville derivatives over the range of the fractional transform. To take the fractional derivatives of $1 / r$ we use the Weyl derivative over the range $\left(R_{0}, \infty\right)$. The Weyl derivative of $f(r)$ can be written as

$$
D^{\alpha} f(r)=\frac{(-1)^{n-1}}{\Gamma(n-\alpha)} \int_{r}^{\infty} \frac{d^{n} f(t)}{d t^{n}}(t-r)^{n-\alpha} d t,
$$

where $n$ is the smallest integer above $\alpha$ when it is fractional. This paper is concerned with the fractional derivative across the boundary and the phase $(-1)^{n-1}$, was chosen to make $D^{\alpha}$ continuous across $\alpha=1$. Applying the derivative definition to $1 / r$ for $\alpha \leqslant 1(n=1)$ we find

$$
D^{\alpha} r^{-1}=-r^{-(1+\alpha)} \Gamma(1+\alpha), \quad \alpha \leqslant 1 .
$$

For $\alpha=1$, this gives the usual first derivative of $1 / r$. For $\alpha>1(n=2)$ the derivative is the same. One should be careful not to interpret the derivative for $\alpha=2$ as the second derivative. The second derivative would follow from a double application of $D^{\alpha}$. For this function the single derivative at $\alpha=2$ is not the same as the double application of the derivative operator.

\section{APPENDIX B: FLUID SPHERE FORMALISM}

Consider the general static spherical metric over the interior fluid with $\nu(r)$ and $\lambda(r)$, 


$$
d s^{2}=-e^{\nu} d t^{2}+e^{\lambda} d r^{2}+r^{2} d \Omega^{2} .
$$

With Einstein's field equations as $G_{i j}=8 \pi T_{i j}$, the energy-momentum components are

$$
\begin{gathered}
8 \pi T_{0}^{0}=-e^{-\lambda}\left(\lambda^{\prime} / r-1 / r^{2}\right)-1 / r^{2}, \\
8 \pi T_{r}^{r}=e^{-\lambda}\left(\nu^{\prime} / r+1 / r^{2}\right)-1 / r^{2}, \\
8 \pi T_{\theta}^{\theta}=8 \pi T_{\phi}^{\phi}=\left(e^{-\lambda} / 2\right)\left[\nu^{\nu^{\prime \prime}}+\left(\nu^{\prime} / 2+1 / r\right)\left(\nu^{\prime}-\lambda^{\prime}\right)\right] .
\end{gathered}
$$

For the fluid interior, the energy-momentum, with four-velocity $U^{i}=\left(e^{\nu / 2}, 0,0,0\right)$, is

$$
T^{i j}=(\rho+P) U^{i} U^{j}+P g^{i j} .
$$

In the comoving frame the fluid stress energy is

$$
T_{0}^{0}=-\rho, \quad T_{r}^{r}=T_{\theta}^{\theta}=T_{\phi}^{\phi}=P .
$$

It is common to use the function $m(r)$ in $g_{r r}$ with

$$
e^{\lambda}=[1-2 m(r) / r]^{-1}
$$

so that

$$
\lambda^{\prime}=2\left(m^{\prime} / r-m / r^{2}\right)(1-2 m / r)^{-1} .
$$

From the first field equation we have

$$
m^{\prime}=4 \pi r^{2} \rho .
$$

The second field equation provides a relation between the fluid pressure, $P$, and $\nu^{\prime}$,

$$
\nu^{\prime} / 2=\left(4 \pi r P+m / r^{2}\right)(1-2 m / r)^{-1}
$$

${ }^{1}$ W. Israel, Phys. Rev. D 15, 935 (1977).

${ }^{2}$ E. Poisson, A Relativist's Toolkit (Cambridge University Press, Cambridge, 2004) p. 89.

${ }^{3}$ W. B. Bonner and P. A. Vickers, Gen. Relativ. Gravit. 13, 29 (1981).

${ }_{5}^{4}$ K. B. Oldham and J. Spanier, The Fractional Calculus (Academic, New York, 1974).

${ }^{5}$ I. M. Sokolov, J. Klafter, and A. Blumen, Phys. Today 55(11), 48 (2002).

${ }^{6}$ C. W. Misner and H. S. Zapolsky, Phys. Rev. Lett. 12, 635 (1964).

${ }^{7}$ C. W. Misner and H. S. Zapolsky, Phys. Rev. Lett. 13, 122 (1964).

${ }^{8}$ R. C. Tolman, Phys. Rev. 55, 364 (1939).

${ }^{9}$ R. Hilfer, Applications of Fractional Calculus in Physics (World Scientific Press, Singapore, 2000), p. 87.

${ }^{10}$ F. Mainardi, Fractals and Fractional Calculus in Continium Mechanics (Springer-Verlag, New York, 1997), p. 291.

${ }^{11}$ M. M. Meerschaert, D. A. Benson, and B. Bäumer, Phys. Rev. E 59, 5026 (1999).

${ }^{12}$ B. Bäumer, M. M. Meerschaert, and J. Mortensen, Proc. Am. Math. Soc. 133, 2273 (2005).

${ }^{13}$ J. Ibañez and J. L. Sanz, J. Math. Phys. 23, 1364 (1982).

${ }^{14}$ H. A. Buchdahl, Phys. Rev. 116, 1027 (1959).

${ }^{15}$ G. Caporaso and K. Brecher, Ann. N.Y. Acad. Sci. 302, 471 (1977).

${ }^{16}$ G. Baym and F. K. Lamb, Neutron Stars, arXiv:physics/0503245, 2005.

${ }^{17}$ R. Adler, J. Math. Phys. 15, 727 (1974).

${ }^{18}$ M. S. R. Delgaty and K. Lake, Comput. Phys. Commun. 115, 395 (1998).

${ }^{19}$ I. Podlubny, Fractional Differential Equations (Academic, New York, 1999).

${ }^{20}$ P. Allegrini, P. Grigolini, and B. J. West, Phys. Rev. E 54, 4760 (1996).

${ }^{21}$ J. P. Krisch, J. Math. Phys. 46, 042506 (2005).

${ }^{22}$ R. M. Wald, General Relativity (University of Chicago Press, Chicago, 1984). 